\title{
An evaluation of the adequacy of pharmaceutical services for the provision of antiretroviral treatment in primary health care clinics
}

\author{
Talitha Crowley*, Ethelwynn L. Stellenberg ${ }^{1}$ \\ Division of Nursing, Stellenbosch University, P.O. Box 19063, Tygerberg, 7500, South Africa
}

\section{A R T I C L E I N F O}

Article history:

Received 21 February 2015

Accepted 26 February 2015

Available online 29 September 2015

Keywords:

Pharmaceutical services

Standards

Primary health care

Antiretroviral therapy

\begin{abstract}
A B S T R A C T
Background: With the introduction of nurse-initiated and -managed antiretroviral therapy (NIMART), new challenges have emerged with regard to the prescribing and dispensing of ART by nurses. One of the key challenges is ensuring adequate pharmaceutical services at PHC clinics.

Objective: The objective of the study was to evaluate the adequacy of pharmaceutical services for the provision of ART in PHC clinics.

Method: A quantitative descriptive study was undertaken in 20 (43\%) randomly selected, eligible clinics in the uMgungundlovu district of KwaZulu-Natal, South Africa.

Results: Clinics used allocated medicine rooms for storing medication, as there were no pharmacies. Problems identified were: insufficient storage space $(50 \% ; n=10)$; inadequate security $(40 \% ; n=8)$; poor air conditioning $(20 \% ; n=4)$, and functional stock-outs of essential drugs $(80 \% ; n=16)$. Professional nurses performed the tasks of managing drug supply and prescribing and dispensing medication as there were no pharmacists or pharmacist's assistants in these clinics.

Conclusion: Human resource constraints necessitate professional nurses to manage drug supplies and to prescribe and dispense medication in resource-constrained PHC clinics. Clear guidelines tailored for PHC are needed to assist nurses in maintaining pharmaceutical service standards when ART services are decentralised.

Copyright ( 2 2015, The Authors. Production and hosting by Elsevier B.V. on behalf of Johannesburg University. This is an open access article under the CC BY-NC-ND license (http://creativecommons.org/licenses/by-nc-nd/4.0/).
\end{abstract}

\footnotetext{
* Corresponding author. Tel.: +27 21 9389036, +27 069453993 (mobile).

E-mail addresses: tcrowley@sun.ac.za (T. Crowley), elstel@sun.ac.za (Ethelwynn L. Stellenberg).

${ }^{1}$ Tel.: +27 21 9389036, +27 829696574 (mobile).
}

Peer review under responsibility of Johannesburg University. http://dx.doi.org/10.1016/j.hsag.2015.02.004

1025-9848/Copyright () 2015, The Authors. Production and hosting by Elsevier B.V. on behalf of Johannesburg University. This is an open access article under the CC BY-NC-ND license (http://creativecommons.org/licenses/by-nc-nd/4.0/). 


\section{Introduction}

\subsection{Background}

South Africa has one pharmacist per 4332 people compared to the minimum country average of one pharmacist per 2300 people recommended by the World Health Organization (WHO) (King \& Fomundam, 2010). This has implications for the safe provision of chronic drug treatment, especially antiretroviral therapy (ART). The shortage of pharmacists in the public health sector in South Africa has led to pharmacists' assistants and nurses taking on drug supply management and dispensing duties to support the ART roll-out (Foster \& McIntyre, 2012).

High-quality, effective health services depend on the timely employment of the right people with appropriate skills at the right time and place (Gilbert, 2013). Yet South Africa has a chronic shortage of trained health care workers, which is exacerbated by disparities between provinces and between the rural and urban sectors (Gilbert, 2013). Subsequently, strategies such as task shifting are welcomed as an alternative, innovative way to alleviate the burden of care in resource-constrained settings (Callaghan, Ford, \& Schneider, 2010).

Task shifting is the process whereby specific tasks are moved appropriately to health workers with less qualifications and shorter training (WHO, 2008). This is not a new concept and has been occurring for decades (Callaghan et al., 2010). The restructuring of health services in South Africa post 1994 and the adoption of a primary healthcare (PHC) approach have placed nurses at the frontline of improving access to PHC services, especially in resource-constrained settings. The need to amend the relevant South African legislation to enable nurses to provide comprehensive health services at a PHC level arose in the 1970s with the establishment of "independent" and self-governing "homelands" (Armstrong et al., 2013). The Nursing Act (No 50 of 1978) was subsequently amended through the promulgation of Section 38A of the Nursing Amendment Act (No 71 of 1981). Registered nurses in the service of the national Department of Health, the provincial administration, a local authority or an organisation delivering a health service designated by the Director-General of Health were authorised to conduct a physical examination; diagnose a physical defect; and keep, supply, administer or prescribe medicine according to the set conditions. In circumstances where nurses are the only healthcare providers at a particular service point, Section 22A(15) of the Medicines and Related Substances Control Act (in terms of the Amendment Act No 90 of 1997) provides for a concession that allows persons or institutions to apply for a permit to acquire, possess, use and supply medication.

The role of nurses was further expanded in 2010 when the Department of Health allowed (under Section 56(6) of the new Nursing Act 33 of 2005) nurses trained in nurse-initiated and -managed antiretroviral treatment (NIMART) to prescribe ART. NIMART is the process whereby appropriately trained nurses assess and initiate new patients to ART, re-prescribe ART for stable patients and refer patients to a physician when needed (Georgeu et al., 2012). Although nurses can be authorised to prescribe and dispense ART, Gray (2010) argues that safe and effective prescribing and dispensing is possible only if it is based on demonstrated competence, and that Section 56(6) should be seen as only a transitional concession, not the norm.

\subsection{Problem statement}

South Africa currently has the largest antiretroviral programme globally (Mayosi et al., 2012). The need to decentralise traditionally hospital-based HIV treatment and care services to PHC clinics, where care is primarily nurse-led, has become apparent with an increasing patient volume (Munderi, Grosskurth, Droti, \& Ross, 2012).

Initiation of ART by professional nurses was shown to increase ART uptake in PHC clinics and reduce workload at referral facilities (Nyasulu, Muchiri, Mazwi, \& Ratshefola, 2013). In addition, a meta-analysis by Emdin, Chong, and Millson (2013) showed that care provided by non-physicians may result in reduced loss to follow-up rates. Despite these encouraging outcomes, evidence for NIMART in Africa is still limited and possible barriers to implementation and its implications for the workforce need further investigation (Emdin et al., 2013; Gilbert, 2013; Georgeu et al., 2012). Resource disparities between clinics and local clinic factors may influence the long-term success and quality of a decentralised model of care through NIMART (Uebel, Guise, Georgeu, Colvin, \& Lewin, 2013). It is therefore important to ensure that standards be maintained in PHC clinics, especially pertaining to the provision of ART.

One of the standards for HIV care in PHC facilities is that the facility must have the medication, supplies and equipment necessary for providing effective HIV services (Pleaner, Moleko, \& Sibanyoni, 2008). Several criteria were identified in order to meet this standard, including access to pharmaceutical support services (Pleaner et al., 2008).

Pharmaceutical services include the acquisition, storage and responsible provision of medication, as well as any medication-related care that will achieve optimal patient outcomes and improve a patient's quality of life (King \& Fomundam, 2010). Pharmacies dispensing ART must meet specific prescribed requirements (Pleaner et al., 2008). Consideration should be given to maintaining basic standards such as adequate drug supply management and pharmaceutical care (King \& Fomundam, 2010).

In a survey of nurses trained in NIMART, only $38 \%$ had a qualification in the dispensing of medication (Cameron et al., 2012). This may indicate that many nurses do not have the knowledge to manage drug supplies and ensure drug safety. In addition, understanding pharmacological principles such as pharmacokinetics and pharmacodynamics, including drug interactions and adverse reactions, are essential to good pharmacy practice (Ruud, Srinivas, \& Toverud, 2010). Yet, nurses' and auxiliary staff members' knowledge of pharmacology, as well as the management of adverse drug reactions, was found to be inadequate in the Eastern Cape, South Africa (Ruud, Srinivas, \& Toverud, 2012). This raises concerns with regard to the quality and efficiency of the pharmaceutical services provided by nurses.

Nurses may be legally authorised to prescribe and dispense medications in a PHC setting (Schellack, 2011). However, Gray 
(2010) argues that the safe provision of medicines is grounded in quality assurance mechanisms such as double checks, which means pharmacists or their assistants should dispense nurse-initiated prescriptions.

All healthcare facilities in South Africa should be able to provide comprehensive services to patients living with HIV/ AIDS (South African Department of Health, 2012). However, pharmaceutical services generally are unevenly distributed between various urban and rural settings in South Africa (Sello, Serfontein, Lubbe, \& Dambisya, 2012). It is therefore essential to investigate whether pharmaceutical services in PHC clinics are adequate for the provision of ART.

According to the National Antenatal Sentinel HIV and Syphilis Prevalence Survey (South African Department of Health, 2011), KwaZulu-Natal has the highest estimated HIV prevalence in the general population (24.7\%). The researcher worked as a clinical nurse practitioner in the uMgungundlovu district at the time of the study and identified several problems related to pharmaceutical services in PHC clinics.

\subsection{Purpose of the study}

The purpose of the study was to determine whether pharmaceutical services were adequate for the provision of ART in $\mathrm{PHC}$ clinics in the uMgungundlovu district of KwaZulu-Natal.

\subsection{Significance}

The benefit of providing HIV treatment and care services at a PHC level is increasingly being acknowledged (Kredo, Ford, \& Garner, 2012). It is unclear, however, if PHC clinics have the necessary resources to deliver the required services and if they are maintaining service standards. Identifying the gaps in pharmaceutical services could lead to strategies to improve the drug supply management and pharmaceutical care in PHC clinics, leading to better outcomes for patients living with HIV as well as for those living with other chronic conditions.

\section{Research method and design}

\subsection{Design}

A cross-sectional descriptive design was applied with a quantitative research approach.

\subsection{Setting}

The study was conducted in PHC clinics in the uMgungundlovu district of KwaZulu-Natal, South Africa.

\subsection{Sampling}

The target population for the purpose of this study was the provincial clinics and local government clinics in the uMgungundlovu district. Clinics had to be operational at least five days a week, $8 \mathrm{~h}$ a day, and had to render comprehensive PHC services from a permanent location. Community healthcare $(\mathrm{CHC})$ facilities were excluded from the study, as these facilities are usually larger and have more resources. A simple random sample of 20 (43\%) clinics was selected to ensure that it was representative. Numbers were assigned to each of the 47 eligible clinics on the list obtained from the district office, and 20 numbers were drawn randomly from a box by a person who was uninvolved in the study.

\subsection{Instrument}

The study formed part of a larger study that evaluated HIV services in PHC clinics. The instrument was developed based on WHO indicators for monitoring and evaluating national antiretroviral programmes (WHO, 2005), South African HIV standards for PHC facilities (Pleaner et al., 2008), and the National Drug Policy for South Africa, (1996). The instrument was divided into Section A (for recording clinic demographic data) and Section B (for recording whether the clinic met the standard criteria). For the main study, several standards were investigated, such as recording and reporting, physical space, basic HIV services rendered, human resources, laboratory services, and pharmaceutical services.

Criteria were measured by assigning a score of 3 if all requirements were met and a score between 0 and 2 if none or some of the requirements were met. For the purpose of evaluating the pharmaceutical services, the data was collapsed into dichotomous categories to determine whether the standard was fully adhered to or not. The standards for pharmaceutical services comprised criteria such as a pharmacy at the facility, adherence to standard operating procedures (SOPs), adequate security, sufficient storage space, and the availability of essential drugs.

\subsection{Pilot study}

The researcher conducted a pilot study in two clinics to ensure the feasibility of the data collection procedure and to test and establish the reliability and validity of the instrument. Data from the pilot study was not included in the main study.

\subsection{Data collection}

District management and clinics were provided with introductory letters to explain the purpose of the study, the data collection procedure and the provisional dates for data collection before clinic visits. The researcher completed the instrument by means of structured observation of clinic data records, infrastructure and processes from December 2008 to January 2009. Clinic managers were consulted when relevant information could not be found.

\subsection{Data analysis}

Data was entered on Microsoft Excel and analysed by a statistical analysing program, STATISTICA (Release 8). A qualified statistician assisted with the data analysis. Descriptive statistics were used to describe the data. Variables such as clinic demographic data and standard criteria were compared by applying non-parametric tests for smaller sample sizes, such as Fisher's test and the Mann-Whitney U test. For some variables, too little variation was present to conduct meaningful analysis. Therefore, only variables that displayed 
sufficient variation were tested for associations. The guideline for determining significant relationships was a $5 \%$ significance level $(p<0.05)$.

\subsection{Ethical considerations}

Ethical approval for the study was obtained from the Ethics Committee of the Faculty of Medicine and Health Sciences at Stellenbosch University (N08/09/237). Permission to conduct the research was obtained from the KwaZulu-Natal provincial research committee, the uMgungundlovu district manager and local municipality managers. Interruption of clinic procedures was limited, since the researcher obtained most information by means of observation. Written, informed consent was obtained from clinic managers before data collection. Data collection instruments were coded and stored in a secure location. Access to raw data was limited to the researcher, statistician and study supervisor.

\section{Trustworthiness}

\subsection{Reliability and validity}

The content and face validity of the instrument was ensured by consulting an expert on HIV research, an expert on nursing research and a qualified statistician. A pilot study was conducted before the main study was undertaken. The researcher adhered to the research protocol and followed the same procedure for data collection at all clinics. Detailed notes were made of observations to ensure that the instrument was completed accurately. Cronbach alpha reliability was not determined, since the data was collapsed into dichotomous categories.

\section{Results}

The results include demographic clinic data and study findings on the adequacy of pharmacy services in the relevant clinics.

\subsection{Demographic data}

The sample of clinics comprised 12 (60\%) provincial and eight (40\%) local government clinics. Most of the clinics $(65 \% ; n=13)$ were open for $8 \mathrm{~h}$ a day on weekdays. Non-governmental organisations (NGOs) employed staff for the ART programme in eight $(40 \%)$ clinics. Ten clinics $(50 \%)$ provided chronic combination ART to patients.

\subsection{Clinic staff and functions}

As shown in Table 1, the clinics were primarily nurse-led. Most clinics (55\%; $\mathrm{n}=11$ ) had only one visiting medical practitioner, while three clinics $(15 \%)$ had no visiting medical practitioner. Only one clinic $(5 \%)$ had a pharmacist visiting once a week.

As shown in Table 2, professional nurses performed the prescription and dispensing of medication. Enrolled nurses
Table 1 - Category and number of staff members $(n=20)$.

\begin{tabular}{|c|c|c|c|c|c|c|}
\hline \multirow{2}{*}{\multicolumn{3}{|c|}{ Category }} & \multicolumn{4}{|c|}{ Number } \\
\hline & & & 0 & 1 & 2 & 3 \\
\hline \multicolumn{3}{|c|}{ Medical practitioner } & $3(15)$ & $11(55)$ & $5(25)$ & $1(5)$ \\
\hline \multicolumn{3}{|c|}{ Enrolled nurse assistant } & $16(80)$ & $3(15)$ & $1(5)$ & 0 \\
\hline \multicolumn{3}{|c|}{ Pharmacist } & $19(95)$ & $1(5)$ & 0 & 0 \\
\hline \multicolumn{3}{|c|}{ Administrative clerk/Support officer } & $2(10)$ & $14(70)$ & $5(25)$ & $1(5)$ \\
\hline \multicolumn{3}{|l|}{ Data capturer } & $14(70)$ & $3(15)$ & $3(15)$ & 0 \\
\hline \multirow[t]{2}{*}{ Category } & \multicolumn{6}{|c|}{ Number } \\
\hline & 1 & 2 & 3 & 4 & 5 & 6 \\
\hline Lay counsellor & $5(25)$ & $9(45)$ & $4(20)$ & 0 & $1(5)$ & $1(5)$ \\
\hline Category & n Mean & $\begin{array}{l}\text { Standar } \\
\text { deviatio }\end{array}$ & \multicolumn{4}{|c|}{ Minimum Maximum } \\
\hline $\begin{array}{l}\text { Professional } \\
\text { nurse }\end{array}$ & $20 \quad 5.75$ & 3.67 & & 2.0 & 13 & \\
\hline $\begin{array}{c}\text { Enrolled } \\
\text { nurse }\end{array}$ & $20 \quad 2.95$ & 1.88 & & 0 & 6 & \\
\hline
\end{tabular}

assisted with the dispensing of chronic pre-packed medication. Administrative clerks or support officers assisted professional nurses with ordering medication, preparing chronic prescriptions for the pharmacy, storing medication in medicine rooms and replenishing drug stock in consultation rooms.

\subsection{Storage of medication}

Most clinics (95\%; $n=19$ ) used allocated medicine rooms for storing medication. One clinic (5\%) did not have an allocated medicine room and stored all drugs in cabinets in consultation rooms (Table 3).

All the clinics dispensed Schedule 5 drugs, which should be locked away securely and recorded in a register. However, only 12 clinics (60\%) had adequate security for medicine rooms, and these rooms were generally accessible to more than one person at the clinic, including non-professional staff members. Statistical analysis revealed no significant difference with regard to medicine room security between local and provincial government clinics (Fisher's exact $p=0.39$ ). However, it was observed that clinics with more staff members were more likely to have better security, although the difference was not significant (Mann-Whitney U p =0.07) (Table 4).

Only half $(50 \% ; n=10)$ of the clinics had sufficient storage space in the medicine room at current volumes. Boxes containing medication were stacked on the floor and medicine rooms were used to store medical and cleaning supplies. There were no significant difference in terms of sufficiency of medicine room storage space between clinics where ART was provided and clinics where ART was not provided (Fisher's exact $p=0.33$ ). Clinics that provided ART generally had larger medicine rooms and therefore had more space for drug and supply storage.

Cold storage is essential, as certain antiretroviral drugs require storage in an air-conditioned room, while vaccines need to be refrigerated. In four clinics $(20 \%)$, the rooms where medication was kept were not air-conditioned. All clinics had a refrigerator for medication, and cold chain management was in place and used correctly in 19 clinics (95\%). 
Table 2 - Gategory and duty of staff members.

\begin{tabular}{ll} 
Category & \multicolumn{1}{c}{ Duty } \\
\hline Medical doctor & Review chronic patients and difficult cases. \\
Professional nurse & Vital signs, minor ailments, integrated management of childhood \\
& illnesses (IMCI), antenatal care (ANC), prevention of mother-to-child \\
& transmission of HIV (PMTCT), family planning (FP), taking of bloods, \\
& injections, prophylaxis (wellness services), PAP smears, emergencies, \\
& immunizations, dressings, counselling, record keeping, TB, chronic care, \\
& HIV tests, health education, prescribing medication, drug supply \\
& management, dispensing, facility administration and facility statistics. \\
& Vital signs, visual screening, TB, dressings, immunizations, FP follow-up, \\
& chronic follow-up, dispensing pre-packed chronic medication and taking \\
& of bloods. \\
Enrolled nurse & Vital signs, dressings and administrative tasks where there are no \\
& administrative clerks. \\
& HIV counselling and testing, PMTCT counselling, ongoing counselling, \\
& health education, ART literacy classes, booking of appointments. \\
Enrolled nurse assistant & Dispensing medication. \\
Lay counsellor & Help desk, reception, opening files, giving patient cards, identifying ill \\
patients, answering the telephone, filing laboratory results, patient & appointments, patient attendance register, stocktaking and equipment \\
Administrative clerk/Support officer & inventory, ordering medication, preparation of chronic prescriptions for \\
& the pharmacy, replenishing stock and medication in consultation rooms, \\
supervising cleaning staff and ordering stationery. & TB register and statistics, filing results of ART patients, completion of \\
ART literacy forms, phoning hospital for patient results, booking patients \\
for hospital visits.
\end{tabular}

\subsection{Standard operating procedures}

None of the clinics had written standard operating procedures (SOPs) for drug receiving, storing and dispensing. However, in most of the clinics medication was stored on shelves with stock cards that were updated as medication was received and dispensed. Although expired drugs were returned to the depots, this was not documented because of the apparent high turnover of drugs in the clinics. According to the SOP, all dispensed medicines should be appropriately labelled with patient and clinic details, as well as instructions for administration. Clinics

Table 3 - Pharmaceutical services $(n=20)$.

\begin{tabular}{lll} 
Criterion & \multicolumn{2}{c}{$\mathrm{n}(\%)$} \\
\cline { 2 - 3 } & \multicolumn{1}{c}{ Yes } & \multicolumn{1}{c}{ No } \\
\hline The facility has its own pharmacy & 0 & $20(100 \%)$ \\
Facility uses a medicine room & $19(95 \%)$ & $1(5 \%)$ \\
Standard operating procedures & 0 & $20(100 \%)$ \\
$\quad$ available and followed & & \\
Electronic ordering of medication & 0 & $20(100 \%)$ \\
Adequate security for medicine room & $12(60 \%)$ & $8(40 \%)$ \\
Sufficient storage space in medicine & $10(50 \%)$ & $10(50 \%)$ \\
$\quad$ room for current volumes & & \\
Dispensing of Schedule 5 drugs & $20(100 \%)$ & 0 \\
Cold storage of drugs & $16(80 \%)$ & $4(20 \%)$ \\
Cold chain management & $19(95 \%)$ & $1(5 \%)$ \\
Registered pharmacist on site & $1(5 \%)$ & $19(95 \%)$ \\
Professional nurse licensed to & $19(95 \%)$ & $1(5 \%)$ \\
$\quad$ dispense on site & & \\
Currently stocking ARV medication & $19(95 \%)$ & $1(5 \%)$ \\
Buffer stock for at least 2 weeks & 0 & $20(100 \%)$ \\
Essential drugs available & $20(100 \%)$ & 0 \\
No functional stock-outs & $2(10 \%)$ & $18(90 \%)$ \\
\hline
\end{tabular}

had printed labels for drugs, but it was impossible to monitor whether these labels were used continuously, as professional nurses dispensed medication from their consultation rooms. None of the clinics ordered medication electronically, even though 13 clinics (65\%) had computers. None of the clinics had electronic pharmacy records.

\subsection{Dispensing}

Although one clinic (5\%) had a registered pharmacist on site for one day a week, the pharmacist did not have a dedicated room and used the data capturers' room, which was unsuitable for drug dispensing. Most clinics $(95 \% ; n=19)$ had at least one professional nurse with a dispensing licence on site, but all the nurses dispensed medication from their consultation rooms. The dispensing practice of individual nurses in their consultation rooms was not monitored in order to ensure patient privacy. Enrolled nurses or other staff members assisted with the dispensing of pre-packed ART at two clinics (10\%).

\subsection{Essential drugs}

Essential drugs (except fluconazole) for treating opportunistic infections were available at all clinics. Fluconazole (used to treat oesophageal candidiasis and cryptococcal meningitis) is doctor-initiated and was therefore unavailable at the clinics. The clinics did not have buffer stock of essential drugs for two weeks. Buffer stock was, however, difficult to determine owing to the lack of documentation, variable patient loads and apparent delays in the arrival of medication orders. Functional stock-out of a certain medicine or nutritional supplement would mean that it was unavailable to be issued to 
Table 4 - Statistical tests $(n=20)$.

\begin{tabular}{|c|c|c|c|c|}
\hline \multirow[t]{3}{*}{ Variables } & \multicolumn{4}{|c|}{ p-values } \\
\hline & \multicolumn{3}{|c|}{ Fisher's exact } & \multirow{2}{*}{$\frac{\text { Mann-Whitney U }}{\text { Total number of staff }}$} \\
\hline & $\begin{array}{c}\text { Local or provincial government } \\
\text { clinic }\end{array}$ & ART available & NGO support & \\
\hline Adequate security for medicine room & 0.39 & 0.68 & 0.62 & 0.07 \\
\hline $\begin{array}{l}\text { Sufficient storage space in medicine room } \\
\text { for current volumes }\end{array}$ & 0.33 & 0.33 & 0.32 & 0.76 \\
\hline
\end{tabular}

patients. As no reports on stock-outs could be found, clinic managers were consulted. In only $10 \%(n=2)$ of the clinics, clinic managers reported no functional stock-outs of essential drugs and nutritional supplements.

\subsection{Antiretroviral therapy (ART)}

Antiretroviral drugs (zidovudine and nevirapine) were available in 19 clinics (95\%) as part of the programme for the prevention of mother-to-child transmission (PMTCT) of HIV. Zidovudine and nevirapine were the drugs used for the PMTCT programme (option A) at the time of the study. The first-line regimen for adults who qualified for combination ART included the drugs stavudine, lamivudine and efavirenz.

Clinics dispensing chronic combination ART $(50 \% ; n=10)$ for stable patients received drugs pre-packed from hospital pharmacies on a weekly basis. This was reported as problematic owing to the time required to prepare and send prescription charts to hospital pharmacies, and insufficient space to store pre-packed medication.

\section{Discussion}

The key finding of the study was that the pharmaceutical services in all the assessed PHC clinics were inadequate according to the basic HIV standards for PHC facilities. None of the clinics complied with all the standard criteria. Standards are important for defining and monitoring the quality of HIV care provided at PHC facilities. These standards provide a framework to assess current services, identify gaps and implement interventions towards improvement (Pleaner et al., 2008).

Before NIMART was introduced and the ART roll-out was expanded in 2010, facilities needed to be accredited before they could provide ART (Pleaner et al., 2008). This accreditation process hindered the expansion of the ART programme. Although currently facilities do not need formal accreditation to provide ART, they should still strive to comply with the basic standards. As adequate pharmaceutical services are central to the expanded provision of ART, pharmaceutical services at PHC clinics should be strengthened to ensure the quality of the services rendered.

In this study, nurses were the main providers of pharmaceutical services, and performed drug supply management as well as the prescription and dispensing of medication. Research has shown that some patients prefer receiving medication from the nurse in the consultation room because of the fear of being stigmatised and identified should they receive ART at the pharmacy (Foster \& McIntyre, 2012). However, the quality control of drugs could be compromised when the prescriber and dispenser are the same person (Gray, 2010). Moreover, nurses' knowledge of the contraindications and side effects of drugs, including the management of adverse drug reaction, may be inadequate to ensure the safety of patients (Cameron et al., 2012; Ruud et al., 2012). Nurses who care for patients on ART should receive training in adverse drug reaction reporting and management (Ruud et al., 2010).

Currently, there is limited evidence on how many nurses are trained and licensed to dispense medication. Cameron et al. (2012) found that only $38 \%$ of NIMART qualified nurses were trained to dispense medication. Many PHC clinics do not have pharmacists or pharmacist's assistants on site, as observed in this study. While 19 clinics (95\%) in this study had at least one nurse licensed to dispense medication on site, nurses dispensed medication from their consultation rooms. It is important that all nurses who dispense medication have a dispensing licence (Schellack, 2011). In addition, clear guidelines should be provided for keeping and dispensing ART stock from consultation rooms.

Adopting additional tasks such as medicine stock management and dispensing could increase the burden on nurses. As nurses remain accountable for their own acts and omissions, they must be adequately supported to deliver the health services for which they are responsible. Clarity concerning the legislation that allows prescribing and dispensing practices by nurses, including a definition of the required competencies, are urgently needed. The roll-out of the 2013 PMTCT guidelines requires nurses working in basic antenatal care to prescribe and dispense fixed-dose combination ART to all HIVpositive pregnant women. However, according to national requirements, ART should be dispensed under the direct supervision of a qualified pharmacist (Georgeu et al., 2012; Gilbert, 2013). The Pharmacy Council of South Africa (2011) clearly describes the scope of practice of pharmacy support staff such as pharmacist's assistants, pharmacy technical assistants and pharmacy technicians, yet the scope of practice of nurses trained in drug dispensing is not clearly outlined.

The role of other support staff, e.g. enrolled nurses and administrative clerks, in the management of drug supplies and the dispensing of medication could be explored further. According to Section 22A(4) (a) of the Medicines and Related Substances Control Act (No 101 of 1965), a practitioner, nurse or person registered under the Health Professions Act of 1979 may compound and dispense a scheduled substance only if they hold a licence, and such a licence will only be issued upon completion of a prescribed course. In terms of Section 56(6) of the Nursing Act (No 33 of 2005), only a nurse (professional 
nurse, midwife or staff nurse) who performs a designated heath service and who has been authorised by the DirectorGeneral may keep and supply medicines. Legally, no person other than a pharmacist, pharmacy support staff, a person with a dispensing licence or a nurse authorised by the Director-General is allowed to handle pharmaceutical stock (National Drug Policy for South Africa, 1996).

The category "staff nurse" will replace the enrolled nurse, and staff nurses may be authorised to perform certain tasks such as the keeping and supply of drugs (Armstrong et al., 2013). However, administrative clerks and lay health workers are unregulated and therefore not allowed to perform tasks related to the handling of drugs. In some settings, prescriptions are pre-packed centrally for patients specified by name. Georgeu et al. (2012) have argued that, in the case of named pre-packed drugs, any lay person could "dispense" the drugs packet to the patient. However, the definition of "dispensing" includes the provision of patient education and assessment of patient adherence (Schellack, 2011). The person who performs this task therefore needs adequate training (National Drug Policy for South Africa, 1996). Tasks should be shifted in a rational way according to the risks involved in the tasks concerned. If tasks are shifted to unregulated workers, clear reporting and accountability lines need to be defined (Armstrong et al., 2013).

Infrastructural challenges such as inadequate storage space, lack of security and the absence of written SOPs were some of the problems identified in this study. The Streamlining Tasks and Roles to Expand Treatment and Care for HIV (STRETCH) trial in the Free State similarly found that the implementation of HIV services in PHC clinics introduced drug storage and management challenges in smaller clinics where the space allocated in their pharmacy was already insufficient (Georgeu et al., 2012; Uebel et al., 2013).

In clinics that provide chronic combination ART, medications were pre-packed centrally by a pharmacist for patients specified by name, and issued by nurses at the clinics. In the STRETCH trial, it was reported that the pre-packing of drugs was problematic, since the pre-packed drugs had to be transported between facilities. Poor communication and transport problems made the continuous supply of ART unreliable (Georgeu et al., 2012). Ordering medications for patients on chronic combination ART will become more complex to handle when the patient load increases significantly. In addition, clear procedures for the ordering and storing of antiretroviral drugs need to be in place if antiretroviral stock is decentralised to PHC clinics.

\section{Limitations of the study}

Results cannot be generalised to other settings, since the study was conducted in one district only. Owing to time and resource constraints, not all clinics in the district could be included. The study did not include a formal pharmacy audit but rather a rapid appraisal of pharmacy adequacy. In addition, observation of the prescription and dispensing practices of professional nurses in consultation rooms could have enhanced the results. The sample size was too small to conduct meaningful statistical analysis on many of the variables.

\section{Recommendations}

It is recommended that all PHC clinics that provide HIV treatment and care services (especially those providing ART) should be formally audited to ensure capacity for such services as regards staff training and infrastructure. The training and placement of pharmacists or pharmacy support staff in every PHC clinic is of the utmost importance to ensure that pharmaceutical standards be maintained.

This study was conducted before the escalated ART rollout in 2010. It has been found that HIV resources may strengthen the larger PHC system (Pfeiffer et al., 2010). Moreover, it should be borne in mind that the uMgungundlovu district is currently one of the pilot districts of the National Health Insurance (NHI) programme (Massyn et al., 2013). A follow-up study could therefore be valuable to determine whether resources for HIV treatment and care services in PHC clinics have improved with these interventions.

\section{Conclusion}

The study has found that the pharmaceutical services in PHC clinics were inadequate to support the current and future HIV treatment and care services with regard to infrastructure, SOPs and trained staff.

Human resource constraints necessitate professional nurses to manage medicine supply and dispense medication in certain PHC clinics. Clear directives and guidelines tailored to PHC clinics are urgently needed to ensure that standards of quality be maintained with regard to the acquisition, storage and supply of medication. Even if nurse models for pharmaceutical care reduce health system costs, this may lay a further burden on nurses and take them away from patient care, eventually compromising the quality of both patient care and medicine control at clinics.

\section{R E F E R E N C E S}

Armstrong, S., Bhengu, B., Kotzé, W., Nkonzo-Mtembu, L., Ricks, E., Stellenberg, E., et al. (2013). A new approach to professional practice. Lansdowne: Juta.

Callaghan, M., Ford, N., \& Schneider, H. (2010). A systematic review of task-shifting for HIV treatment and care in Africa. Human Resources for Health, 8(8). Retrieved from http://www. human-resources-health.com/content/8/1/8.

Cameron, D., Gerber, A., Mbatha, M., Mutyabule, J., Swart, H., \& West, N. (2012). Nurse initiation and maintenance of patients on antiretroviral therapy: are nurses in primary care clinics initiating ART after attending NIMART training? South African Medical Journal, 102(2), 98-100.

Emdin, C., Chong, N. J., \& Millson, P. E. (2013). Non-physician clinician provided HIV treatment results in equivalent outcomes as physician-provided care: a meta-analysis. Journal of the International AIDS Society, 16(18445). Retrieved from http://dx.doi.org/10.7448/IAS.16.1.18445.

Foster, N., \& McIntyre, D. (2012). Economic evaluation of taskshifting approaches to the dispensing of anti-retroviral therapy. Human Resources for Health, 10(1)(32), 1-10. Retrieved from http://www.human-resources-health.com/content/10/1/32. 
Georgeu, D., Colvin, C. J., Lewin, S., Fairall, L., Bachman, M. O., ..., et al. (2012). Implementing nurse-initiated and managed antiretroviral treatment (NIMART) in South Africa: a qualitative process evaluation of the STRETCH trial. Implementation Science, 7(66). Retrieved from http://www. implementationscience.com/content/7/1/66.

Gilbert, L. (2013). "Re-engineering the workforce to meet service needs": exploring "task-shifting" in South Africa in the context of HIV/AIDS and antiretroviral therapy. South African Review of Sociology, 44(2), 54-75. Retrieved from http://www. tandfonline.com/doi/abs/10.1080/21528586.-2013.802537.

Gray, A. (2010). Prescribing and dispensing by nurses: neglected steps in the legislative process. HIV Nursing Matters Magazine, 1, 28-31.

King, R. C., \& Fomundam, H. N. (2010). Remodeling pharmaceutical care in sub-saharan africa (SSA) amidst human resources challenges and the hiv/aids pandemic. International Journal Health Planning and Management, 25, 30-48.

Kredo, T., Ford, N., \& Garner, P. (2012). Decentralising HIV treatment delivery in middle- and low- income countries. Cochrane Database of Systematic Reviews, 2012(7), 1-8.

Massyn, N., Day, C., Dombo, M., Barron, P., English, R., \& Padarath, A. (2013). District health barometer 2012/13. Health Systems Trust. Retrieved from http://www.hst.org.za.

Mayosi, B. M., Lawn, J. E., van Niekerk, A., Bradshaw, D., Abdool Karim, S. S., \& Coovadia, H. M. (2012). Health in South Africa: changes and challenges since 2009. Lancet, 380(9858), 2029-2043.

Munderi, P., Grosskurth, H., Droti, B., \& Ross, D. A. (2012). What are the essential components of HIV treatment and care services in low- and middle-income countries: an overview by settings and levels of the health system? AIDS, 26(2), 97-103.

National Drug Policy for South Africa. (1996). Retrieved from http://apps.who.int/medicinedocs/en/d/-Js17744en/.

Nyasulu, J. C. Y., Muchiri, E., Mazwi, S., \& Ratshefola, M. (2013). NIMART rollout to primary health care facilities increases access to antiretrovirals in Johannesburg: an interrupted time series analysis. South African Medical Journal, 103(4), 232-236.

Pfeiffer, J., Montoya, P., Baptista, A. J., Karagianis, M., Pugas, M. D. M., ..., et al. (2010). Integration of hiv/aids services into african primary health care: lessons learned for health system strengthening in Mozambique - a case study. Journal of the International AIDS Society, 13(3). Retrieved from http://www. jiasociety.org/content/12/1/3.

Pharmacy Council of South Africa. (2011). Scope of practice, supervision of pharmacy support personnel and qualifications. Government Gazette, 34428(1), 16-29.

Pleaner, M., Moleko, W., \& Sibanyoni, M. (2008). HIV standards: A self-assessment tool to improve HIV services at primary health care facilities. University of the Witwatersrand. Reproductive Health and HIV Institute (RHRU).

Ruud, K. W., Srinivas, S. C., \& Toverud, E. (2010). Addressing gaps in pharmacovigilance practices in the antiretroviral therapy program in the eastern cape Province, South Africa. Research in Social and Administrative Pharmacy, 6(4), 345-353.

Ruud, K. W., Srinivas, S. C., \& Toverud, E. (2012). Healthcare providers' experiences with adverse drug reactions and adherence challenges in antiretroviral therapy of HIV patients in the eastern cape Province, South Africa. European Journal of Clinical Pharmacology, 68, 1321-1328.

Schellack, G. (2011). Nursing pharmacology and medicine management: Part 1 pharmacology in clinical Practice. Professional Nurse Today, 15(1), 35-43.

Sello, D. A., Serfontein, J. H. P., Lubbe, M. S., \& Dambisya, Y. M. (2012). Factors influencing access to pharmaceutical services in underserviced areas of the west rand District, gauteng Province, South Africa. Health SA Gesondheid, 17(1). Retrieved from http://www.hsag.co.za/index.php/HSAG-/article/view/ 609.

South African Department of Health. (2011). The national antenatal Sentinel HIV and syphilis prevalence survey, South Africa, 2011.

South African Department of Health. (2012). National strategic plan on HIV, STIs and TB, 2012-2016.

Uebel, K., Guise, A., Georgeu, D., Colvin, C., \& Lewin, S. (2013). Integrating HIV care into nurse-led primary health care services in South Africa: a synthesis of three linked qualitative studies. BMC Health Services Research, 13, 171. Retrieved from http://www.biomedcentral.com/content/1472-6963/12/171.

World Health Organization. (2005). National AIDS programmes: A guide to indicators for monitoring and evaluating national antiretroviral programmes.

World Health Organization. (2008). Task Shifting: Global recommendations and guidelines. 